\title{
A Selection-Mutation Model for Q-learning in Multi-Agent Systems
}

\author{
Karl Tuyls ${ }^{*}$ \\ Computational Modeling Lab \\ Pleinlaan 2 \\ 1050 Brussels, Belgium \\ ktuyls@vub.ac.be
}

\author{
Katja Verbeeck \\ Computational Modeling Lab \\ Pleinlaan 2 \\ 1050 Brussels, Belgium \\ kaverbee@vub.ac.be
}

\author{
Tom Lenaerts \\ Computational Modeling Lab \\ Pleinlaan 2 \\ 1050 Brussels, Belgium \\ tlenaert@vub.ac.be
}

\begin{abstract}
Although well understood in the single-agent framework, the use of traditional reinforcement learning (RL) algorithms in multi-agent systems (MAS) is not always justified. The feedback an agent experiences in a MAS, is usually influenced by the other agents present in the system. Multi agent environments are therefore non-stationary and convergence and optimality guarantees of RL algorithms are lost.

To better understand the dynamics of traditional RL algorithms we analyze the learning process in terms of evolutionary dynamics. More specifically we show how the Replicator Dynamics (RD) can be used as a model for Q-learning in games. The dynamical equations of Q-learning are derived and illustrated by some well chosen experiments. Both reveal an interesting connection between the exploitationexploration scheme from RL and the selection-mutation mechanisms from evolutionary game theory.
\end{abstract}

\section{Categories and Subject Descriptors}

I.2 [Artificial Intelligence]: Learning

\section{General Terms}

Theory, Experimentation

\section{Keywords}

Reinforcement Learning, Multi-Agent Systems, Replicator Dynamics

\section{INTRODUCTION}

Reinforcement Learning ( RL) is already an established and profound theoretical framework for learning in stand-

\footnotetext{
*Author is a Ph.d. student, funded by a doctoral grant of the institute for advancement of scientific technological research in Flanders(IWT)
}

Permission to make digital or hard copies of all or part of this work for personal or classroom use is granted without fee provided that copies are not made or distributed for profit or commercial advantage and that copies bear this notice and the full citation on the first page. To copy otherwise, to republish, to post on servers or to redistribute to lists, requires prior specific permission and/or a fee.

AAMAS'03, July 14-18, 2003, Melbourne, Australia.

Copyright 2003 ACM 1-58113-683-8/03/0007 ...\$5.00. alone systems. Yet, extending RL to multi-agent systems (MAS) does not guarantee the same theoretical grounding. As long as the environment an agent is experiencing is stationary, and the agent can experiment enough, RL guarantees convergence to the optimal strategy. In a MAS however, the reinforcement an agent receives, may depend on the actions taken by the other agents, present in the system.

Consider for instance the problem of finding the optimal way between two points in traffic. The cost measured in time it takes to get from point $\mathrm{A}$ to a point $\mathrm{B}$ using a particular route will be influenced by the current traffic conditions, i.e. how many other drivers decided to use the same route. Communications on these decisions is not always possible, moreover there is an associated cost and is subject to delays. Uncontrolled exploration in this situations can lead to policy oscillations, [?]. When everyone decides to take the alternative route, this one becomes less interesting than the original one. Most MAS systems belong to this last case of non-stationarity. Obviously in these environments, the convergence results of RL are lost.

In the light of the above problem it is important to fully understand the dynamics of reinforcement learning and the effect of exploration in MAS. For this aim we review the Replicator Dynamics (RD) model from Evolutionary Game Theory (EGT). This model consists of a system of differential equations and plays a central role in biological and economical models.

Several authors have already noticed that the RD can emerge from simple learning models, $[1$, ?]. In [?] a learning automata model was used. In this paper the dynamics, which emerges from Q-learning agents, are analyzed in 2player games. A version of the RD corrected with an additional term is found. This extra term, which can be interpreted as an entropy term reveals an interesting connection between the exploitation-exploration schemes from RL and the selection-mutation mechanisms from EGT. The dynamics we derived, opens new perspectives in understanding and fine tuning the learning process in MAS.

The outline of the paper is as follows. In the next section we briefly introduce Q-learning in games and the replicator equations from EGT. In section 3 we derive the dynamical equations of Q-learning, in which we recognize the RD. In section 4 the derived equations are discussed in both domains, e.g. RL and EGT. We continue with an illustration of the results of section 3 with some well chosen games in section 5. Finally we conclude in the last section. 


\section{BACKGROUND}

This section provides some background in learning in games and the replicator dynamics. Game theory, [5] offers the mathematical foundation for the analysis and synthesis of problems in decentralized control. A game consists of more than one player and results in an outcome for every player depending on the overall behavior of all the players. Formally it can be described by a tuple $\left(n, A_{1} \ldots A_{n}, R_{1} \ldots R_{n}\right)$ where $n$ is the number of players, $A_{i}$ the set of actions available to player $i$ and $R_{i}: A_{1} \times \ldots \times A_{n} \rightarrow \Re$ player $i$ his payoff function. When games are played repeatedly a sequential decision problem with one state arises. In this paper players are modeled as Q-learners, which is described in the following subsection.

\subsection{Q-learning in games}

A model of reinforcement learning consists of: a discrete set of environment states, a discrete set of agent actions and a set of scalar reinforcement signals. On each step of interaction the agent receives a reinforcement and some indication of the current state of the environment, and chooses an action. The agent's job is to find a policy, i.e. a mapping from states to actions, that maximizes some long-run measure of reinforcement.

Common reinforcement learning methods, which can be found in [10] are structured around estimating value functions. A value of a state or state-action pair, is the total amount of reward an agent can expect to accumulate over the future, starting from that state. One way to find the optimal policy is to find the optimal value function. If a perfect model of the environment as a Markov decision process is known, the optimal value function can be learned with an algorithm called value iteration. Q-learning is an adaptive value iteration method see [?], which bootstraps its estimate for the state-action value $Q_{t+1}(s, a)$ at time $t+1$ upon its estimate for $Q_{t}\left(s^{\prime}, a^{\prime}\right)$ with $s^{\prime}$ the state where the learner arrives after taking action $a$ in state $s$ :

$Q_{t+1}(s, a) \leftarrow(1-\alpha) Q_{t}(s, a)+\alpha\left(r+\gamma \max _{a^{\prime}} Q_{t}\left(s^{\prime}, a^{\prime}\right)\right)$

With $\alpha$ the usual step size parameter, $\gamma$ a discount factor and $r$ the immediate reinforcement.

The players could therefore use the algorithm of (1) where the state information $s$ is removed. Solutions are formulated in terms of equilibrium situations for the players.

\subsection{The Replicator Equations}

The basic concepts and techniques developed in EGT were initially formulated in the context of evolutionary biology. $[14, ?]$. In this context, the strategies of all the players are genetically encoded (called genotype). Each genotype refers to a particular behavior which is used to calculate the payoff of the player. The payoff of each player's genotype is determined by the frequency of other player types in the environment.

One way in which EGT proceeds is by constructing a dynamic process in which the proportions of various strategies in a population evolve. Examining the expected value of this process gives an approximation which is called the RD. An abstraction of an evolutionary process usually combines two basic elements: selection and mutation. Selection favors some varieties over others, while mutation provides variety in the population. RD highlights the role of selection, it describes how systems consisting of different strategies change over time. They are formalized as a system of differential equations. Each replicator (or genotype) represents one (pure) strategy $P_{j}(n)$ (or $\left.Q_{j}(n)\right)$. This strategy is inherited by all the offspring of the replicator. The general form of a replicator dynamic is the following:

$$
\frac{d x_{i}}{d t}=\left[(A \mathbf{x})_{i}-\mathbf{x} \cdot A \mathbf{x}\right] x_{i}
$$

In equation (2), $x_{i}$ represents the density of strategy $i$ in the population, $A$ is the payoff matrix which describes the different payoff values each individual replicator receives when interacting with other replicators in the population. The state of the population $(\mathbf{x})$ can be described as a probability vector $\mathbf{x}=\left(x_{1}, x_{2}, \ldots, x_{J}\right)$ which expresses the different densities of all the different types of replicators in the population. Hence $(A \mathbf{x})_{i}$ is the payoff which replicator $i$ receives in a population with state $x$ and $\mathbf{x} \cdot A \mathbf{x}$ describes the average payoff in the population. The growth rate $\frac{\frac{d x_{i}}{d t}}{x_{i}}$ of the population share using strategy $i$ equals the difference between the strategy's current payoff and the average payoff in the population. For further information we refer the reader to $[14, ?]$.

In this paper the players are Q-learners. We consider a game to be played between the members of two different populations, each population representing one Q-learner. As a result, we need two systems of differential equations: one for the row player $(P)$ and one for the column player $(Q)$. This setup corresponds to a $\mathrm{RD}$ for asymmetric games. If $A=B^{t}$, equation (2) would again emerge.

This translates into the following replicator equations for the two populations:

$$
\begin{aligned}
& \frac{d p_{i}}{d t}=\left[(A \mathbf{q})_{i}-\mathbf{p} \cdot A \mathbf{q}\right] p_{i} \\
& \frac{d q_{i}}{d t}=\left[(B \mathbf{p})_{i}-\mathbf{q} \cdot B \mathbf{p}\right] q_{i}
\end{aligned}
$$

As can be seen in equation (3) and (4), the growth rate of the types in each population is now determined by the composition of the other population. Note that, when calculating the rate of change using these systems of differential equations, two different payoff matrices $(A$ and $B)$ are used for the two different players.

\section{THE Q-LEARNING DYNAMICS}

In this section we derive mathematically ${ }^{1}$ the relation between Q-learning and the RD (see sections 2.1 and 2.2). More precisely we construct a continuous time limit of the Q-learning model, where Q-values are interpreted as Boltzmann probabilities for the action selection. For simplicity we consider games between 2 players. Each agent(or player) has a probability vector over his action set, more precisely $x_{1}, \ldots, x_{n}$ over action set $a_{1}, \ldots, a_{n}$ for the first player and $y_{1}, \ldots, y_{m}$ over $b_{1}, \ldots, b_{m}$ for the second player. Formally the Boltzmann distribution is described by,

$$
x_{i}(k)=\frac{e^{\tau Q_{a_{i}}(k)}}{\sum_{j=1}^{n} e^{\tau Q_{a_{j}}(k)}}
$$

\footnotetext{
${ }^{1}$ The reader who is interested in the complete derivation, we refer to our technical report [?]
} 
where $x_{i}(k)$ is the probability of playing strategy $i$ at time step $k$ and $\tau$ is the temperature.

Now we can find an expression for, $x_{i}(k+1)$.

$$
\begin{aligned}
\frac{x_{i}(k+1)}{x_{i}(k)} & =\frac{e^{\tau Q_{a_{i}}(k+1)} \sum_{j} e^{\tau Q_{a_{j}}(k)}}{e^{\tau Q_{a_{i}}(k)} \sum_{j} e^{\tau Q_{a_{j}}(k+1)}} \\
& =\frac{e^{\tau Q_{a_{i}}(k+1)} e^{-\tau Q_{a_{i}}(k)} \sum_{j} e^{\tau Q_{a_{j}}(k)}}{\sum_{j} e^{\tau Q_{a_{j}}(k+1)}} \\
& =\frac{e^{\tau \Delta Q_{a_{i}}(k)}}{\sum_{j} x_{j} e^{\tau \Delta Q_{a_{j}}(k)}}
\end{aligned}
$$

From which follows,

$$
x_{i}(k+1)=x_{i}(k) \frac{e^{\tau \Delta Q_{a_{i}}(k)}}{\sum_{j} x_{j} e^{\tau \Delta a_{j}(k)}}
$$

If we consider the difference equation for $x_{i}$ we have,

$$
\begin{aligned}
x_{i}(k+1)-x_{i}(k) & =\frac{x_{i}(k) e^{\tau \Delta Q_{a_{i}}(k)}}{\sum_{j} x_{j}(k) e^{\tau \Delta Q_{a_{j}}(k)}}-x_{i}(k) \\
& =x_{i}(k)\left(\frac{e^{\tau \Delta Q_{a_{i}}(k)}-\sum_{j} x_{j}(k) e^{\tau \Delta Q_{a_{j}}(k)}}{\sum_{j} x_{j}(k) e^{\tau \Delta Q_{a_{j}}(k)}}\right)
\end{aligned}
$$

For the continuous time version we suppose that the amount of time that passes between two repetitions of the game is given by $\delta$ with $0<\delta \leq 1$. The variable $x_{i}(k \delta)$ describes the $\mathrm{x}$-values at time $k \delta=t$. Under this assumptions we have,

$$
\begin{aligned}
\frac{x_{i}(k \delta+\delta)-x_{i}(k \delta)}{\delta}= & \frac{x_{i}(k \delta)}{\delta \sum_{j} x_{j}(k \delta) e^{\tau \Delta Q_{a_{j}}(k \delta)}} \times \\
& \left(e^{\tau \Delta Q_{a_{i}}(k \delta)}-\sum_{j} x_{j}(k \delta) e^{\tau \Delta Q_{a_{j}}(k \delta)}\right)
\end{aligned}
$$

We are interested in the limit with $\delta \rightarrow 0$. We find the state of the limit process at some time $t \geq 0$ (which we keep fixed) by taking the limit of $x_{i}(k \delta)$ with $\delta \rightarrow 0$ and $k \delta \rightarrow t$.

$$
\begin{aligned}
\lim _{\delta \rightarrow 0} \frac{\Delta x_{i}(k \delta)}{\delta}= & \lim _{\delta \rightarrow 0} \frac{x_{i}(k \delta)}{\delta \sum_{j} x_{j}(k \delta) e^{\tau \Delta Q_{a_{j}}(k \delta)}} \times \\
& \left(e^{\tau \Delta Q_{a_{i}}(k \delta)}-\sum_{j} x_{j}(k \delta) e^{\tau \Delta Q_{a_{j}}(k \delta)}\right) \\
= & \lim _{\delta \rightarrow 0} \frac{x_{i}(k \delta)}{\sum_{j} x_{j}(k \delta) e^{\tau \Delta Q_{a_{j}}(k \delta)}} \times \\
& \lim _{\delta \rightarrow 0}\left(\frac{e^{\tau \Delta Q_{a_{i}}(k \delta)}}{\delta}-\frac{\sum_{j} x_{j}(k \delta) e^{\tau \Delta Q_{a_{j}}(k \delta)}}{\delta}\right)
\end{aligned}
$$

The first limit,

$$
\lim _{\delta \rightarrow 0} \frac{x_{i}(k \delta)}{\sum_{j} x_{j}(k \delta) e^{\tau \Delta Q_{a_{j}}(k \delta)}}
$$

equals $x_{i}(t)$ or short $x_{i}$, because the exponent term becomes $1\left(\Delta Q_{a_{j}}(k \delta)\right.$ becomes zero) and $\sum_{j} x_{j}(k \delta)$ equals 1 (sum of all probabilities equals 1 ). Therefore,

$$
\lim _{\delta \rightarrow 0} \frac{\Delta x_{i}(k \delta)}{\delta}=x_{i} \underbrace{\times \lim _{\delta \rightarrow 0}\left(\frac{e^{\tau \Delta Q_{a_{i}}(k \delta)}}{\delta}-\frac{\sum_{j} x_{j}(k \delta) e^{\tau \Delta Q_{a_{j}}(k \delta)}}{\delta}\right)}_{T_{2}}
$$

The second limit is undefined (we have a $\frac{0}{0}$ situation), which allows us to use the rule of the l'hôspital. The second term now equals (for short $T_{2}$ ),

$$
\begin{aligned}
T_{2}= & \lim _{\delta \rightarrow 0} \frac{\tau \Delta Q_{a_{i}}(k \delta) e^{\tau \Delta Q_{a_{i}}(k \delta)}}{\delta}- \\
& \sum_{j} x_{j}(k \delta) \times \lim _{\delta \rightarrow 0}\left(\tau \Delta Q_{a_{j}}(k \delta) \frac{e^{\tau \Delta Q_{a_{j}}(k \delta)}}{\delta}\right) \\
= & \tau \frac{d Q_{a_{i}}(t)}{d t}-\sum_{j} x_{j}(t) \tau \frac{d Q_{a_{j}}(t)}{d t}
\end{aligned}
$$

The total limit now becomes,

$$
\frac{\frac{d x_{i}}{d t}}{x_{i}}=\tau\left(\frac{d Q_{a_{i}}}{d t}-\sum_{j} \frac{d Q_{a_{j}}}{d t} x_{j}\right)
$$

We now have derived the continuous time model of the Qlearning process. As you can see in equation (5) we need an expression for $\frac{d Q_{a_{i}}(t)}{d t}$. We can derive the differential equation for the Q-function by performing the following steps.

The Q-learning update rule for the first player can be written as follows,

$$
Q_{a_{i}}(k+1)=Q_{a_{i}}(k)+\alpha\left(r_{a_{i}}(k+1)+\gamma \max _{a_{i}} Q-Q_{a_{i}}(k)\right)
$$

which implies,

$$
\Delta Q_{a_{i}}(k)=\alpha\left(r_{a_{i}}(k+1)+\gamma \max _{a_{i}} Q-Q_{a_{i}}(k)\right)
$$

This expression is the difference equation for the Q-function. If we make this equation infinitesimal, going from discrete steps to a continuous version, we suppose that the amount of time that passes between two repetitions of updates of the $\mathrm{Q}$-values is given by $\delta$ with $0<\delta \leq 1$. The variable $Q_{a_{i}}(k \delta)$ describes the $\mathrm{Q}$-values at time $k \delta$. Now, we get,

$\Delta Q_{a_{i}}(k \delta)=\alpha\left(r_{a_{i}}((k+1) \delta)+\gamma \max _{a_{i}} Q-Q_{a_{i}}(k \delta)\right)((k+1) \delta-k \delta)$

which is the same as writing,

$$
\Delta Q_{a_{i}}(k \delta)=\alpha\left(r_{a_{i}}((k+1) \delta)+\gamma \max _{a_{i}} Q-Q_{a_{i}}(k \delta)\right) \delta
$$

We are interested in the limit $\delta \rightarrow 0$. We find the state of the limit process at some time $t \geq 0$ (which we keep fixed) by taking the limit of $Q_{a_{i}}(k \delta)$ with $\delta \rightarrow 0$ and $k \delta \rightarrow t$. If we now bring $\delta$ to the left side, and take the limit for $\delta \rightarrow 0$, we have

$$
\frac{d Q_{a_{i}}}{d t}=\alpha\left(r_{a_{i}}+\gamma \max _{a_{i}} Q-Q_{a_{i}}\right)
$$

Now, we can substitute equation (6) in equation (5), yield- 
ing,

$$
\begin{aligned}
\frac{\frac{d x_{i}}{d t}}{x_{i}}= & \tau\left(\alpha r_{a_{i}}+\alpha \gamma \max _{a_{i}} Q_{a_{i}}-\alpha Q_{a_{i}}-\right. \\
& \left.\sum_{j} x_{j} \alpha\left(r_{a_{j}}+\gamma \max _{a_{i}} Q_{a_{i}}-Q_{a_{j}}\right)\right) \\
= & \tau \alpha\left(r_{a_{i}}-\sum_{j} x_{j} r_{a_{j}}-Q_{a_{i}}+\sum_{j} Q_{a_{j}} x_{j}\right)
\end{aligned}
$$

because $\sum_{j} x_{j}=1$, this expression becomes,

$$
\begin{aligned}
\frac{\frac{d x_{i}}{d t}}{x_{i}} & =\tau \alpha\left(r_{a_{i}}-\sum_{j} x_{j} r_{a_{j}}-Q_{a_{i}} \sum_{j} x_{j}+\sum_{j} Q_{a_{j}} x_{j}\right) \\
& =\tau \alpha\left(r_{a_{i}}-\sum_{j} x_{j} r_{a_{j}}+\sum_{j} x_{j}\left(Q_{a_{j}}-Q_{a_{i}}\right)\right)
\end{aligned}
$$

because $\frac{x_{j}}{x_{i}}$ equals $\frac{e^{\tau Q_{a_{j}}}}{e^{\tau Q_{a_{i}}}}$, we have

$$
\alpha \sum_{j} x_{j} \ln \left(\frac{x_{j}}{x_{i}}\right)=\alpha \tau \sum_{j} x_{j}\left(Q_{a_{j}}-Q_{a_{i}}\right)
$$

which gives us,

$$
\frac{\frac{d x_{i}}{d t}}{x_{i}}=\alpha \tau\left(r_{a_{i}}-\sum_{j} x_{j} r_{a_{j}}\right)+\alpha \sum_{j} x_{j} \ln \left(\frac{x_{j}}{x_{i}}\right)
$$

Now suppose that we have payoff matrices $A$ and $B$ for the 2 players. Now we can write $r_{a_{i}}$ as $\sum_{j} a_{i j} y_{j}$. This results in,

$$
\frac{d x_{i}}{d t}=x_{i} \alpha \tau\left((A \mathbf{y})_{i}-\mathbf{x} \cdot A \mathbf{y}\right)+x_{i} \alpha \sum_{j} x_{j} \ln \left(\frac{x_{j}}{x_{i}}\right)
$$

analogously for the second player, we have,

$$
\frac{d y_{i}}{d t}=y_{i} \alpha \tau\left((B \mathbf{x})_{i}-\mathbf{y} \cdot B \mathbf{x}\right)+y_{i} \alpha \sum_{j} y_{j} \ln \left(\frac{y_{j}}{y_{i}}\right)
$$

Equations 7 and 8 express the dynamics of both Q-learners in terms of Boltzmann probabilities. In the next section we discuss the derived equations, followed by an illustration in the last section.

\section{DISCUSSION}

In this section we analyze the evolutionary model expressed by (7) and (8), both in an evolutionary perspective as in a RL perspective.

\subsection{Selection - Mutation perspective}

Comparing (7) or (8) with the RD in (2), we see that the first term of (7) or (8) is exactly the RD and thus takes care of the selection mechanism, see [14]. The mutation mechanism for Q-learning is therefore left in the second term, i.e.

$$
x_{i} \alpha \sum_{j} x_{j} \ln \left(\frac{x_{j}}{x_{i}}\right)
$$

and can be rewritten as:

$$
x_{i} \alpha\left(\sum_{j} x_{j} \ln \left(x_{j}\right)-\ln \left(x_{i}\right)\right)
$$

In equation (9) we recognize 2 entropy terms, one over the entire probability distribution $x$, and one over strategy $x_{i}$.
We can describe the entropy terms as follows:

$$
S_{i}=-x_{i} \ln \left(x_{i}\right)
$$

and

$$
S_{n}=-\sum_{j} x_{j} \ln \left(x_{j}\right)
$$

$S_{i}$ describes the information we have concerning strategy $i$, $S_{n}$ describes the information concerning the entire distribution. Therefore (9) can be written as

$$
-\left(\alpha x_{i} S_{n}-\alpha S_{i}\right)
$$

In [?] the mutation equation was derived as a difference between the old state of $x_{i}$ and the new one,

$$
\sum_{j} \epsilon_{i j} x_{j}-x_{i}
$$

where $\epsilon_{i j}$ explicitly represents the mutation rate from strategy $j$ to strategy $i$, and $\epsilon_{i j} \geq 0 \forall j=1, \ldots, n$ and $\sum_{j} \epsilon_{i j}=1$. In the Q-learning dynamics mutation is expressed analogously with entropy expressing the state or information of a strategy.

Relating entropy and mutation is not new. It is a well known fact $[8,9]$ that mutation increases entropy. In [9], it is stated that the concepts are familiar with thermodynamics in the following sense: the selection mechanism is analogous to energy and mutation to entropy. So generally speaking, mutations tend to increase entropy. In figure 1 we show the entropy function $S_{n}$ in the $[0,1]$ interval for binomial strategies. Entropy reaches its maximum at 0.5, which is normal because $x$ is then an equiprobable distribution, meaning there is a lot of uncertainty about which strategy will be played.

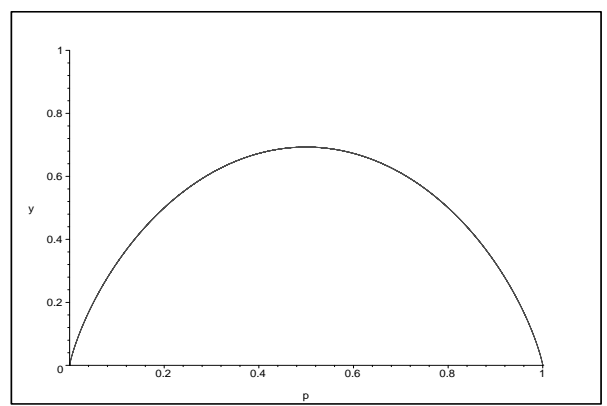

Figure 1: The entropy function for a binomial distribution.

The dynamic equations for Q-learning reveal that selection happens as expressed in a $\mathrm{RD}$, i.e. a strategy is favored according to the pay-off it receives, relative to it's opponent. As seen in the formula a mutation effect is also present. This is calculated by the difference in entropy of a strategy compared to the whole population.

\subsection{Exploration - Exploitation perspective}

One of the great challenges of reinforcement learning is the trade-off between exploration and exploitation. To collect enough reward an agent should select one of the actions which returned a big payoff in the past, but to discover such actions the agent should try actions he didn't try before. Mapping exploration and exploitation to selection 
and mutation we obtain a biological interpretation of the exploration-exploitation concept in RL. More precisely, the replicator dynamics of (7), i.e. the first term

$$
x_{i} \alpha \tau\left((A \mathbf{y})_{i}-\mathbf{x} \cdot A \mathbf{y}\right)
$$

takes care of selecting better strategies over time, which maps perfectly on the concept of exploitation (being greedy). The mutation term of (7), i.e. the second term

$$
x_{i} \alpha \sum_{j} x_{j} \ln \left(x_{j}\right)-\alpha x_{i} \ln \left(x_{i}\right)
$$

increases entropy, but at the same time it provides variety. Exploration can be considered as the mutation concept, as both concepts take care of providing variety. In the dynamic model of RL (see equation 7 ) variation is measured by entropy, in the algorithmic version of RL (see 1) it is a matter of correctly setting the exploration parameter. The exploration-exploitation trade-off is normally a difficult fine tuning process of the necessary parameters in the learning process. With the selection-mutation model of equations (7) and (8), we avoid this problem as illustrated by the experiments in section 5 .

To conclude this discussion, we remark that the mathematical derivation of section 3 will not change if we allow multiple states. We also remark that equations 7 and 8 make explicit that each player takes into account the other player for its immediate reward. This is implicit in RL.

\section{EXPERIMENTS}

In this section we describe some experiments that illustrate the mathematical derivation of section 3 . The experiments have been conducted with $2 \times 2$ games. The general payoff matrices, $A$ for the first player and $B$ for the second, are defined in table 1 . The family of $2 \times 2$ games is usually classified in three subclasses, as follows[6],

$$
\mathrm{A}=\left(\begin{array}{ll}
a_{11} & a_{12} \\
a_{21} & a_{22}
\end{array}\right) \quad \mathrm{B}=\left(\begin{array}{ll}
b_{11} & b_{12} \\
b_{21} & b_{22}
\end{array}\right)
$$

Table 1: The left matrix (A) defines the payoff for the row player, the right matrix (B) defines the payoff for the column player

Subclass 1: if $\left(a_{11}-a_{21}\right)\left(a_{12}-a_{22}\right)>0$ or $\left(b_{11}-b_{12}\right)\left(b_{21}-\right.$ $\left.b_{22}\right)>0$, at least one of the 2 players has a dominant strategy, therefore there is just 1 strict equilibrium.

Subclass 2: if $\left(a_{11}-a_{21}\right)\left(a_{12}-a_{22}\right)<0,\left(b_{11}-b_{12}\right)\left(b_{21}-\right.$ $\left.b_{22}\right)<0$, and $\left(a_{11}-a_{21}\right)\left(b_{11}-b_{12}\right)>0$, there are 2 pure equilibria and 1 mixed equilibrium.

Subclass 3: if $\left(a_{11}-a_{21}\right)\left(a_{12}-a_{22}\right)<0,\left(b_{11}-b_{12}\right)\left(b_{21}-\right.$ $\left.b_{22}\right)<0$, and $\left(a_{11}-a_{21}\right)\left(b_{11}-b_{12}\right)<0$, there is just 1 mixed equilibrium.

The first subclass includes those type of games where each player has a dominant strategy, as for instance the prisoners dilemma. However it includes a larger collection of games since only 1 of the players needs to have a dominant strategy. In the second subclass none of the players has a dominated strategy. But both players receive the highest payoff by both playing their first or second strategy. This is expressed in the condition $\left(a_{11}-a_{21}\right)\left(b_{11}-b_{12}\right)>0$. The third subclass only differs from the second in the fact that the players don't receive their highest payoff by both playing the first or the second strategy. This is expressed by the condition $\left(a_{11}-a_{21}\right)\left(b_{11}-b_{12}\right)<0$. In the following three subsections we describe the results of the experiments conducted in each subclass.

\subsection{Subclass 1}

For this subclass we considered the prisoners dilemma game [?, 14]. In this game both players have the same strategy set containing one dominant strategy, more precisely defect. The payoff matrices for this game are defined as follows,

$$
\mathrm{A}=\left(\begin{array}{ll}
1 & 5 \\
0 & 3
\end{array}\right) \quad \mathrm{B}=\left(\begin{array}{ll}
1 & 0 \\
5 & 3
\end{array}\right)
$$

Table 2: Prisoners dilemma: The left matrix $(A)$ defines the payoff for the row player, the right one $(B)$ for the column player.

In figure 2 the direction field plot of the differential equations of the prisoners dilemma game is plotted. These equations can be derived by filling in the payoff matrices $A$ and $B, \alpha$ and $\tau$ in equations (7) and (8). The x-axis expresses the probability with which player 1 plays strategy 1 (defect), and the $y$-axis expresses the probability with which player 2 plays strategy 1 (defect). As you can see the equations are plotted for 3 values of $\tau$, more precisely 1,2,10. Only in the last one the dynamics attain the Nash equilibrium (the only attractor in the last plot) for the game at the coordinates $(1,1)$. In the first 2 plots $\tau$ is not chosen big enough to reach for the Nash equilibrium.

In figure 3 we also plotted the Q-learning process for the same game with the same settings as for the system of differential equations. As starting points for the Q-learning process we chose a grid of 25 points from which we plotted the results for the following 8 points, $\{(0.1 ; 0.7),(0.3 ; 0.3),(0.3 ; 0.7)$, $(0.5 ; 0.7),(0.7 ; 0.3),(0.7 ; 0.5),(0.7 ; 0.7),(0.9 ; 0.1)\}$. The main reason for this is to keep the plots clear and well-organised. In every point a learning path starts and converges to a particular point. If you compare the plots with the direction field plots for the same value of $\tau$ you can see that the sample paths of the learning process approximates the paths of the differential equations. This allows us to fully understand and predict the Q-learning process with our evolutionary model of equations (7) and (8).

\subsection{Subclass 2}

For the second subclass we considered the battle of the sexes game [?, 14] defined by the payoff matrices of figure 3. In figure 4 the direction field plot of the differential equations of this game is plotted. Again the direction field of the equations are plotted for 3 values of $\tau$, more precisely $1,2,10$. In the first 2 plots $\tau$ isn't big enough to reach for one of the three Nash equilibria. Only in the last one the dynamics attain the Nash equilibria (the 3 attractors in the last plot) for the game at the coordinates $(1,1),(0,0)$ and $\left(\frac{2}{3}, \frac{1}{3}\right)$. The mixed equilibrium though is very unstable. Any small perturbation away from this equilibrium will typically lead the dynamics to one of the 2 pure equilibria.

In figure 5 we also plotted the Q-learning process for the 
same game with the same settings as for the system of differential equations. As starting points for the Q-learning process we chose the same grid of points used for the experiments under subclass 1. Again in every point a learning path starts and converges to a particular point. If you compare the plots with the direction field plots for the same value of $\tau$ you can see that the sample paths of the learning process approximates the paths of the differential equations. The instability of the mixed equilibrium is the reason why this equilibrium doesn't emerge from the learning process.

$$
\mathrm{A}=\left(\begin{array}{ll}
2 & 0 \\
0 & 1
\end{array}\right) \quad \mathrm{B}=\left(\begin{array}{ll}
1 & 0 \\
0 & 2
\end{array}\right)
$$

Table 3: Battle of the sexes: The left matrix $(A)$ defines the payoff for the row player, the right one $(B)$ for the column player.

\subsection{Subclass 3}

The third class consists of the games with a unique mixed equilibrium. For this category we used the game defined by the matrices in table 4. Typical for this class of games is that the interior trajectories define closed orbits around the equilibrium point. You can see this in figure 6.

Also for this experiment we used the same grid of starting points as we used in the previous 2 subclasses. Only in the last plot we only used 3 starting points for reasons of clarity. Again in every point a learning path starts and converges to a particular point. If you compare the plots with the direction field plots for the same value of $\tau$ you can see that the sample paths of the learning process approximates the paths of the differential equations.

$$
\mathrm{A}=\left(\begin{array}{ll}
2 & 3 \\
4 & 1
\end{array}\right) \quad \mathrm{B}=\left(\begin{array}{ll}
3 & 1 \\
2 & 4
\end{array}\right)
$$

Table 4: The left matrix $(A)$ defines the payoff for the row player, the right one $(B)$ for the column player.

\section{CONCLUSIONS}

In this paper we derived a general selection-mutation model of the dynamics of multiple Q-learners playing a game. Using this model an evolutionary interpretation of the explorationexploitation scheme from reinforcement learning is given.

Learning can be very time consuming, especially when you need to fine tune some parameters. As the experiments illustrate, plotting the direction field of the selection-mutation model beforehand gives information on how to initialize the learning agents so that they end up in the most interesting attractors of the game.

In a next step we will apply these ideas to multiple-state and multiple-player games.

\section{REFERENCES}

[1] Börgers, T., Sarin, R., Learning Through Reinforcement and Replicator Dynamics. Journal of Economic Theory, Volume 77, Number 1, November 1997.

[2] Gintis, C.M., Game Theory Evolving. Princeton University Press, June 2000.
[3] Hofbauer, J., Sigmund, K., Evolutionary Games and Population Dynamics, Cambridge University Press, 1998.

[4] A. Nowé and K. Verbeeck, Distributed Reinforcement learning, Loadbased Routing a case study, author="A. Nowé and K. Verbeeck", Notes of the Neural, Symbolic and Reinforcement Methods for sequence Learning Workshop at ijcai99, 1999, Stockholm, Sweden.

[5] Osborne J.O., Rubinstein A., A course in game theory. Cambridge, MA: MIT Press (1994).

[6] Redondo, F.V., Game Theory and Economics, Cambridge University Press, (2001).

[7] L. Samuelson, Evolutionary Games and Equilibrium Selection, MIT Press, Cambridge, MA, 1997.

[8] Schneider, T.D., Evolution of biological information. journal of NAR, volume 28, pages 2794 - 2799, 2000.

[9] Stauffer, D., Life, Love and Death: Models of Biological Reproduction and Aging. Institute for Theoretical physics, Köln, Euroland, 1999.

[10] Sutton, R.S., Barto, A.G. : Reinforcement Learning: An introduction. Cambridge, MA: MIT Press (1998).

[11] Tuyls, K., Lenaerts, T., Verbeeck, K., Maes, S. and Manderick, B, Towards a Relation Between Learning Agents and Evolutionary Dynamics. Proceedings of BNAIC 2002. KU Leuven, Belgium.

[12] Tuyls, K. and Verbeeck, K., The Replicator Dynamics as a model for Q-learning in games. Technical Report CoMo/10/2002, Vrije Universiteit Brussel, Belgium.

[13] Watkins, C. and Dayan, P., Q-learning. Machine Learning, 8(3):279-292, 1992.

[14] Weibull, J.W., Evolutionary Game Theory, MIT Press, (1996). 

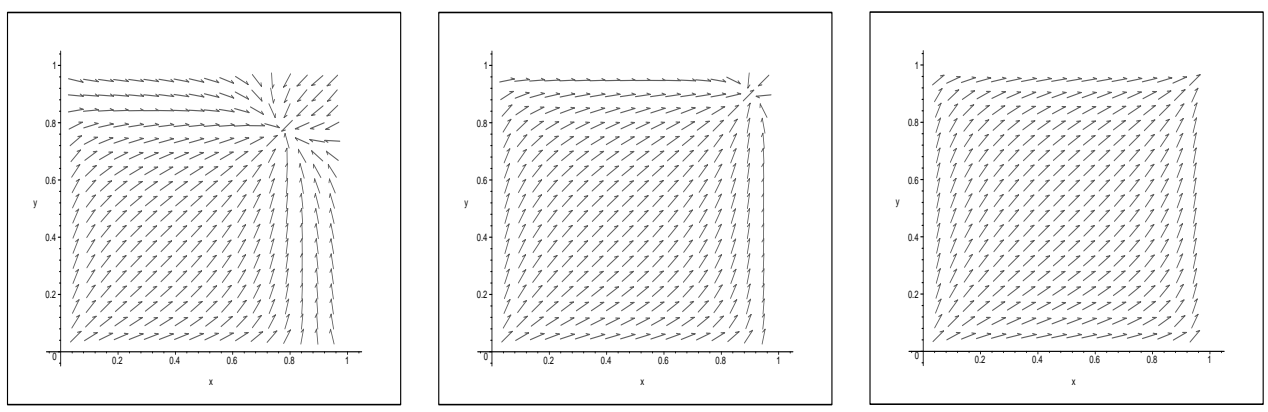

Figure 2: The direction field plots of the prisoners dilemma (subclass 1) game with $\tau=1,2,10$
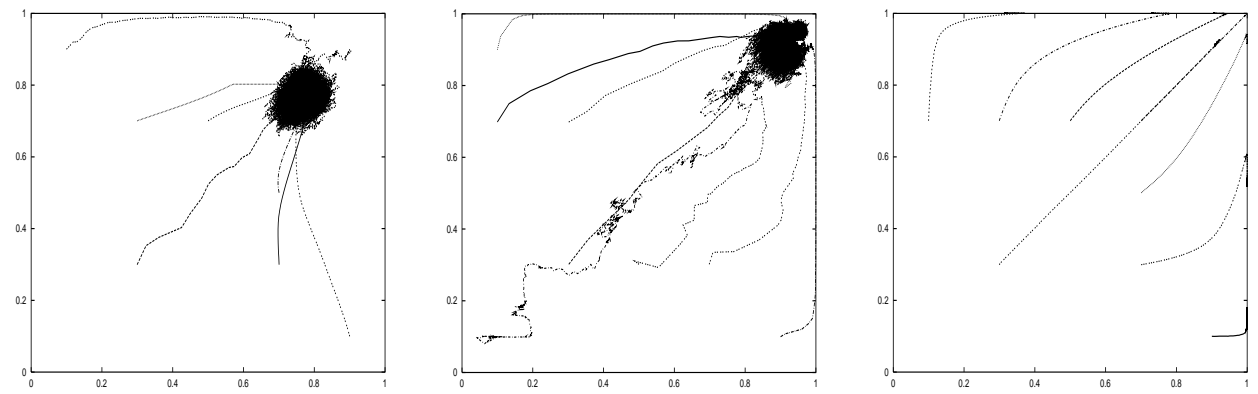

Figure 3: The Q-learning plots of the prisoners dilemma (subclass 1) game with $\tau=1,2,10$
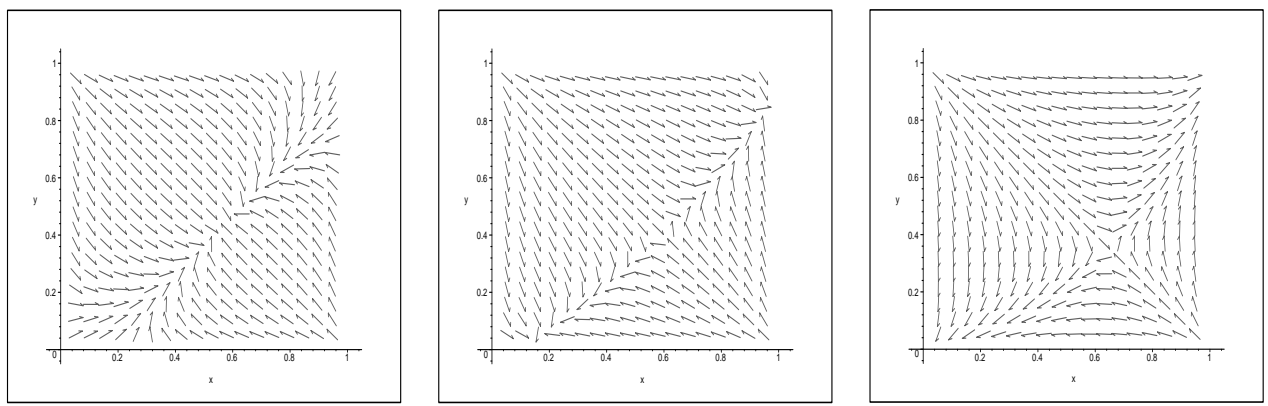

Figure 4: The direction field plots of the battle of the sexes (subclass 2) game with $\tau=1,2,10$
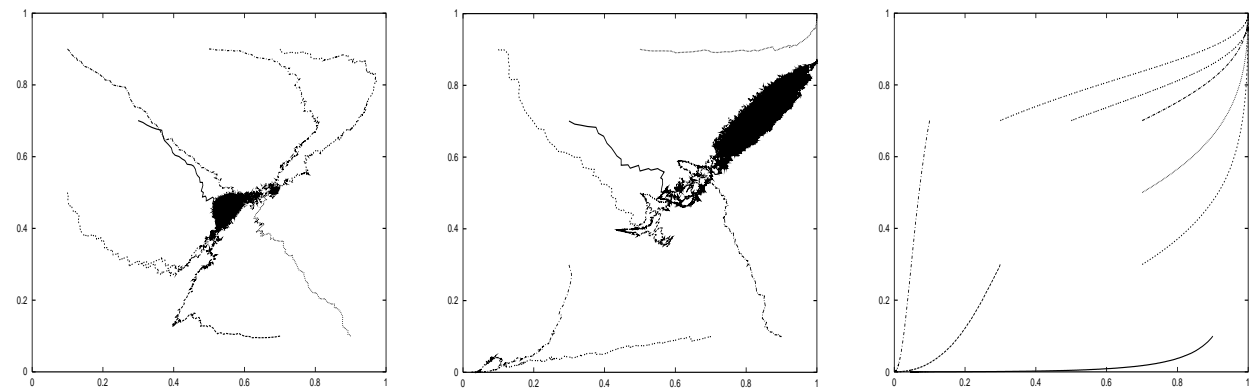

Figure 5: The Q-learning plots of the battle of the sexes (subclass 2) game with $\tau=1,2,10$ 

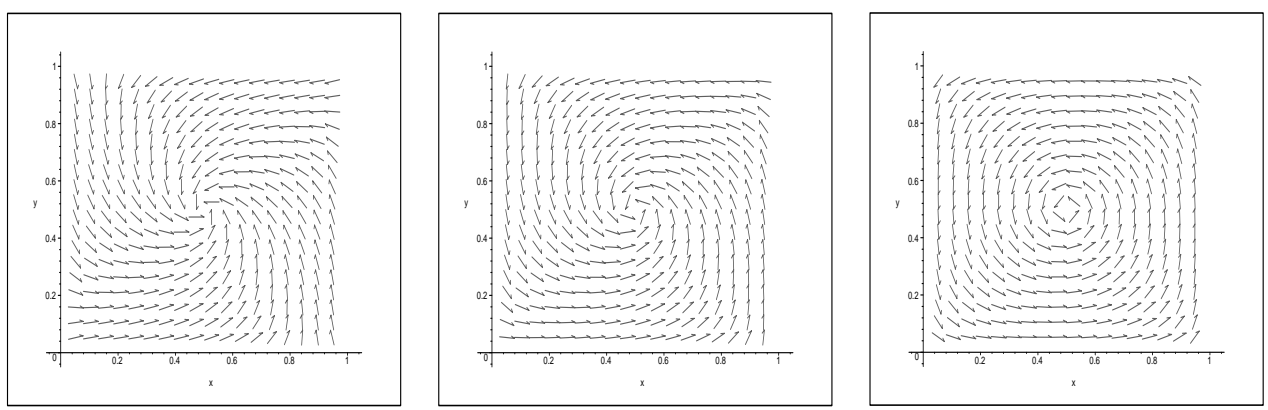

Figure 6: The direction field plots of subclass 3 with $\tau=1,2,10$
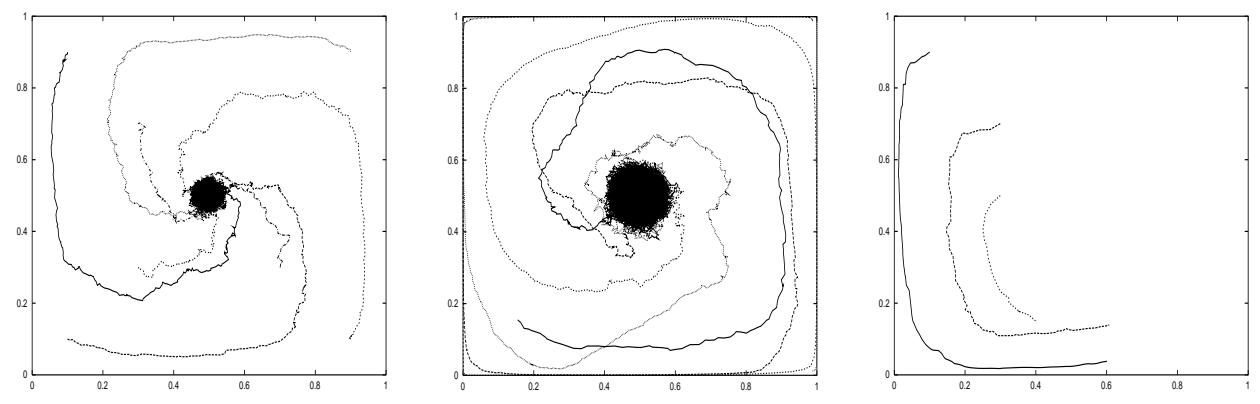

Figure 7: The Q-learning plots of subclass 3 with $\tau=1,2,10$ 